\title{
Publisher Correction: Predicting drug-protein interaction using quasi-visual question answering system
}

Shuangjia Zheng, Yongjian Li, Sheng Chen, Jun Xu (D) and Yuedong Yang (D)

Correction to: Nature Machine Intelligence https://doi.org/10.1038/s42256-020-0152-y, published online 14 February 2020.

In the version of this Article originally published, the placeholder text 'Please add some text here' after the section heading 'Experiments' was mistakenly not updated; this text should have read 'In this section, we will introduce the employed datasets and experiments to indicate the performance of our DrugVQA model'. This has now been corrected.

Published online: 11 August 2020

https://doi.org/10.1038/s42256-020-0224-Z

(C) The Author(s), under exclusive licence to Springer Nature Limited 2020 